\title{
Pulmonary Bone Cement Embolism following Pedicle Screw Instrumentation with Bone Cement Augmentation for a Burst Fracture of L1 in a Non-Osteoporotic Spine
}

\author{
Jose Manuel F. Ignacio ${ }^{1}$ and Sharon D. Ignacio ${ }^{2}$ \\ ${ }^{1}$ Department of Orthopedics, College of Medicine and Philippine General Hospital, University of the Philippines Manila \\ ${ }^{2}$ Department of Rehabilitation Medicine, College of Medicine and Philippine General Hospital, University of the Philippines Manila
}

\begin{abstract}
This is a case of pulmonary cement embolism in a young nonosteoporotic patient who sustained a burst fracture of L1. Bone cement augmentation was done for the fractured vertebra as well as over other segments where pedicle screws were inserted. The procedure eventually led to pulmonary cement embolism. The pathway for cement to reach the pulmonary vasculature from within the vertebral body is shown thru a series of imaging studies with the CT Scan done for the patient showing the route taken by the cement as it proceeds towards the pulmonary artery. This is rarely, if ever, shown in published literature. The literature is reviewed further to provide a rational management strategy for such a potentially life threatening condition.
\end{abstract}

Key Words: pulmonary cement embolism, vertebroplasty, kyphoplasty, cement augmentation of pedicle screw, burst fracture, PMMA (polymethyl methacrylate)

\section{Introduction}

With the advent of vertebroplasty and kyphoplasty, many new uses for bone cement augmentation have further evolved. One such modification is the introduction of bone cement into the vertebral bodies prior to insertion of pedicle screws in order to increase screw pull out strength. ${ }^{1}$ Likewise, bone cement augmentation of traumatic vertebral body fractures have been reported with the aim of rendering the segment stable. ${ }^{2}$ With new technology comes new risks. The advantages of vertebroplasty and kyphoplasty are numerous and the risks are low but potentially life threatening. This report looks into one of the known risks of these procedures and it will attempt to explore what factors are involved in its development. Possible ways to decrease the occurrence of pulmonary cement embolism shall be discussed as well as management principles in the event of its occurrence.

Corresponding author: Jose Manuel F. Ignacio, MD

Spine Section

Department of Orthopedics

Philippine General Hospital

University of the Philippines Manila

Taft Avenue, Ermita, Manila 1000 Philippines

Telephone: +6325548466

Fax No.: +632 5218538

Email: spinedoc222@yahoo.com

\section{Case Presentation}

A 34-year-old seaman was transferred from an overseas hospital for postoperative management and rehabilitation of a spine injury. His history started three weeks prior to admission when, while working on a ship's deck, he fell backwards landing onto a lower deck. He experienced severe back pain and narrates that he could not move his lower extremities. He was transferred from his ship to a hospital in a country nearby. The patient developed bladder and bowel incontinence after the injury although he noted that his lower extremity motor function began returning to his left toes even before his operation which took place three days after his injury. The patient had CT scan and MRI studies done on this admission (Figures 1 and 2).

According to the transfer notes, the surgery performed was described as a T12-L1 cemented arthrodesis as well as an L4-5 laminectomy with bilateral foramenotomy and discectomy. Post-operatively, there appeared to have been a loss of motor function anew but there was gradual recovery thereafter whilst patient was undergoing physical therapy. It was at this point that the patient was transferred to his home country for further physical rehabilitation.

On admission, the patient was noted to be ambulant with a foot drop and with minimal back pain. Physical examination revealed absence of the right ankle motors and toe dorsiflexor. He was bladder and bowel incontinent.

Initial radiographs revealed pedicle screw fixation of T12-L2 with the screws augmented with bone cement. Aside from these findings, there was the ominous presence of serpentine looking opacities at the perivertebral region as well as over the anterior epidural spaces at the L1-2 areas (Figure 3). Harboring a suspicion of bone cement extravasation into the surrounding vascular structures, further examinations were done including a CT scan of the lumbosacral region with coronal, sagittal and 3D reconstruction. A Chest CT scan revealed cement embolus within the pulmonary artery (Figure 4). 


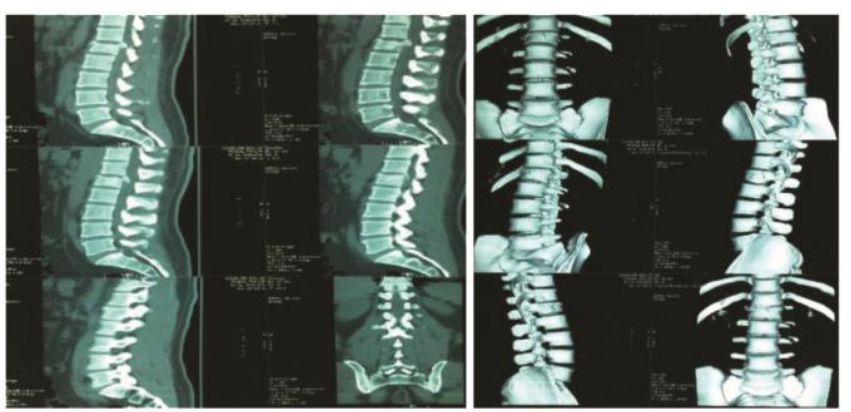

Figure 1. Sagittal and 3D reconstruction of injury studies showing a burst fracture of L1 with a fragment retropulsed into the spinal canal

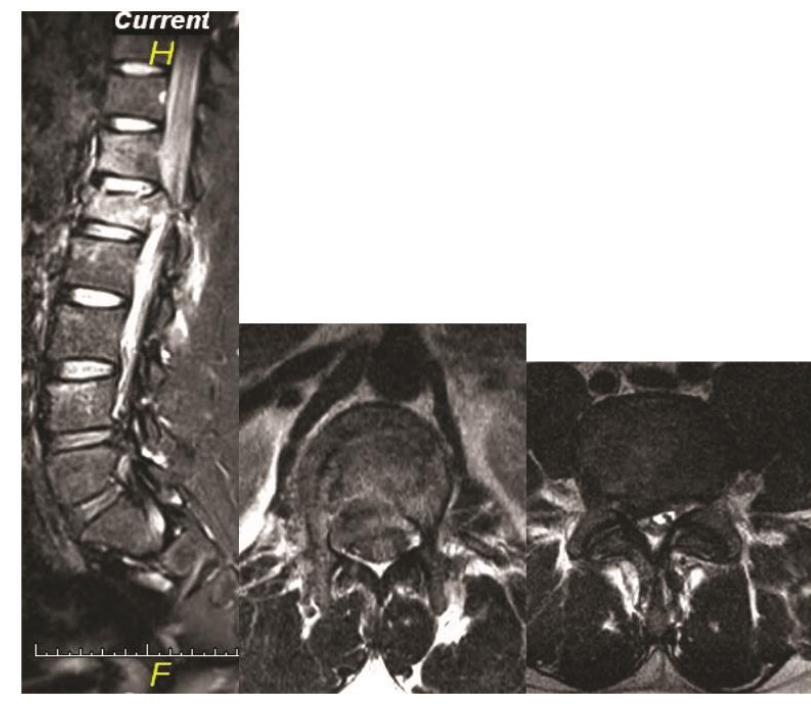

L1

L4-5 disc

Figure 2. MRI image showing the L1 burst fracture and a central and right sided disc herniation at the L4-5 disc

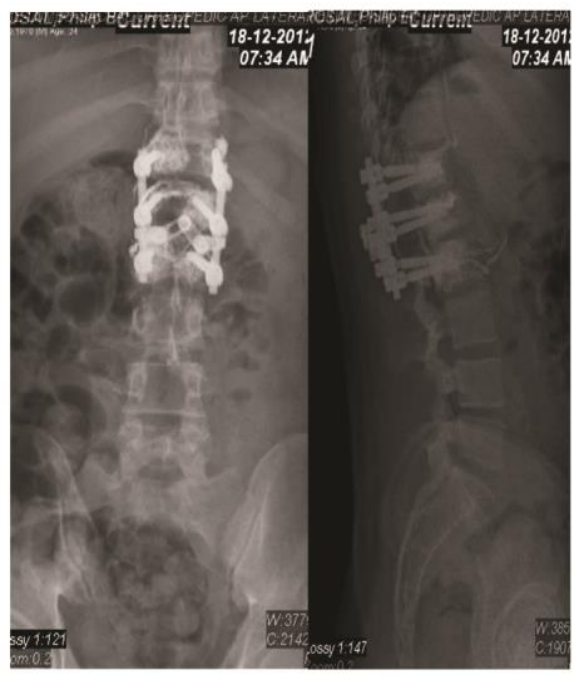

Figure 3. Radiographs done upon admission showing serpentine opacities at the perivertebral areas

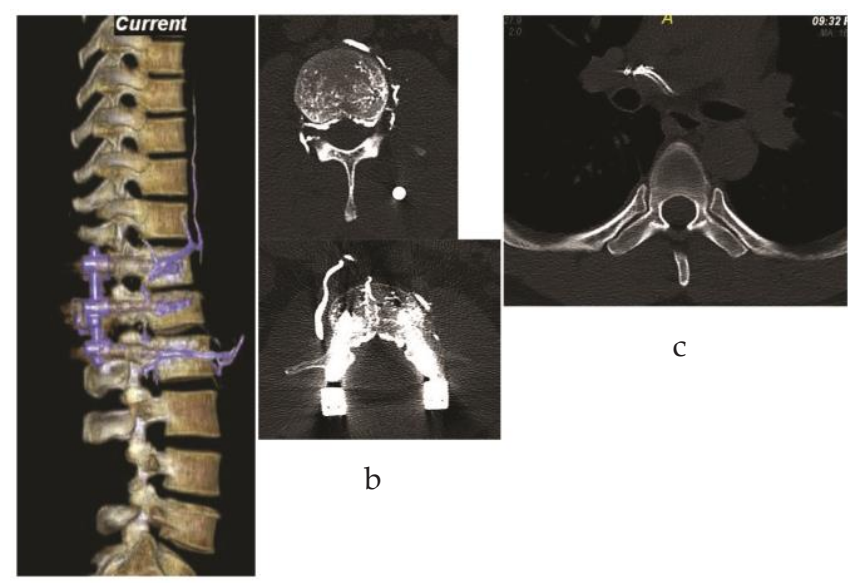

a

Figure 4. CT Scan studies showing (a) cement extravasation around the lumbar spine and the pathway taken by the cement towards the lungs. (b) Axial images showing cement extravasation from within the body onto the perivertebral plexus via the nutrient and epidural vessels. (c) Bone cement lodged at the pulmonary artery

Despite the presence of pulmonary bone cement embolism, the patient did not show signs of respiratory compromise, and 2D echocardiography showed good cardiac function. Since the patient was asymptomatic, he was simply managed with anticoagulants which was planned for a 6 month course. The patient was subsequently discharged able to ambulate with an ankle foot orthosis for his foot drop which failed to recover and on an intermittent catheterization program for his bladder incontinence.

\section{Discussion}

The reported incidence of pulmonary cement embolization following vertebroplasty varies depending on the sensitivity of the examination utilized. A large multicenter prospective RCT (VERTOS II) utilized CT scans to document the presence of pulmonary cement embolism at a mean of 22 months after surgery. ${ }^{3}$ Pulmonary cement embolism was seen in a quarter of the patients tested. All of the patients were asymptomatic. Other studies show a much grimmer picture with percentages reaching $90 \%$ for vertebroplasty ${ }^{4}$ to $37.5 \%$ for balloon kyphoplasty. ${ }^{5}$

Unlike vertebroplasty and kyphoplasty, the risk of developing pulmonary cement embolism following bone cement augmentation of pedicle screws is less than $1 \%$. These procedures are done to increase the pull out strength of the screws in osteoporotic spines thus making the fixation more stable. Further combining the procedure with a vertebroplasty or kyphoplasty increases the risk to around 3 $-23 \% .6,7,8$

Several risk factors were identified enabling the cement from within the vertebral bodies to extravasate and reach the 
lungs. Various studies point to the presence of cement in certain areas of the venous vasculature as being risk factors in developing pulmonary embolus. Unlike the study by Venmans et al., who found a significant risk factor being the presence of cement in the Azygos, ${ }^{3} \mathrm{Kim}$ et al. found that cement leakage into the inferior vena cava was closely related to the development of pulmonary cement embolism. ${ }^{8}$ Another study by Choe et al. showed paravertebral venous cement leakage as being a risk factor to pulmonary cement embolism. ${ }^{9}$

In this patient being presented, the presence of cement was identified in the epidural and paravertebral plexus of veins as well as within the inferior vena cava and the Hemiazygos vein. The presence in one or the other of these sites as being a sole risk factor in pulmonary cement embolism may not be an accurate observation since, as shown in the axial sequences of the CT scan image, the cement actually extravasated from the inner portion of the vertebral body outwards via the nutrient vessel onto the vertebral plexus (Figure $4 \mathrm{a}$ and $\mathrm{b}$ ). The embolus then proceeded onto the inferior vena cava and the Hemiazygos vein thru the heart and eventually, the pulmonary vasculature (Figure 4c). It is believed by the authors of this report that this is the pathway for the cement in reaching the lungs and that the previous studies cited only the parts which were captured in the CT scan images done on their patients. Likewise, cement formation within the vessels as seen in these extensive CT scan 3D images (Figure 4a) do not necessarily form a continuum as it finds its way towards the central vasculature onto the lungs. There will be areas which will be devoid of cement only later to reappear at a more central site. The extensive documentation of this patients' pulmonary cement embolism, made more graphic by $3 \mathrm{D}$ CT scan sequences gives support to the thinking that the pathology and risk factors are not limited to just certain areas where the cement is documented to be present. The mere fact that the cement leaked outside to the paravertebral vasculature means that the cement may already travel towards the lungs.

The dangers of pulmonary embolism may be acute or chronic in nature. Acute symptoms produces severe respiratory dysfunction. Most pulmonary cement embolisms are asymptomatic. ${ }^{8}$ Treatment options include anticoagulant administration for 3-6 months ${ }^{6}$ and surgical removal of the embolus for symptomatic patients.

The importance of the viscosity of the acrylic cement when injected during vertebroplasty was discussed in a case reported by Bernhard et al. ${ }^{10}$ Injection of cement in a very low viscous state will most likely increase the chances of the cement leaking out of the confines of the vertebral body as compared to injecting the cement with a firmer consistency. The importance of using high viscosity cement in decreasing the incidence of venous cement leak has been shown in the study by Alselmetti et al. ${ }^{11}$ In the case which is being presented, performing the vertebroplasty with the aim of augmenting the pullout strength of the screw on the vertebral body was in all probability done with the cement in a more liquid phase so that the cement will not harden prior to insertion of the screws. This is the usual technique when inserting cement prior to inserting screws. This is the reason why fenestrated screws were invented. When using screws with a fenestrated design, the surgeon is allowed to insert the screw first and cement can then be applied onto the head of the screw which then brings the cement into the vertebral body by way of a channel within the screw shaft. In this patient, when the cement was injected in a very liquid state, the stage was set for an easy extravasation of the cement outside the confines of the vertebrae and into the surrounding vascular structures.

Management of such conditions are dependent on the location of the emboli and whether the patient is symptomatic or not. In a systematic review by Kreuger, et al., a decision making tree was proposed. ${ }^{7}$ The factors considered are whether the embolus is peripherally or centrally located and if the patient is symptomatic or not. Asymptomatic peripheral emboli are managed by observation. Anti-coagulation treatment is reserved for symptomatic peripheral or asymptomatic central involvement. Surgery is limited only for the symptomatic central or pulmonary types of cement embolization since anticoagulant therapy will not open an occluded vessel. The purpose for anticoagulant therapy is merely to limit the formation of thrombus around the cement. ${ }^{9}$ The paper by Kreuger also mentions reports where surgeons regretted having opened up their patients with central types of emboli presumably due to the difficulty in performing the surgery and all its attendant risks. ${ }^{7}$

\section{Conclusion}

This case report highlights a complication of vertebroplasty which was previously thought to be rare but which is being shown to be not an infrequent complication after all. Pulmonary cement embolism is usually asymptomatic but is potentially life threatening. A high index of suspicion is warranted and management rests on whether the patient has a peripheral or central type of embolization and whether the patient is symptomatic or not. Based on these parameters, patients can either be observed only, treated with anticoagulants or managed with surgery.

\footnotetext{
References

1. Wuisman PI, Van Dijk M, Staal H, Van Royen BJ. Augmentation of (pedicle) screws with calcium apatite cement in patients with severe progressive osteoporotic spinal deformities: an innovative technique. Eur Spine J. 2000; 9(6):528-33.

2. Afzal S, Akbar S, Dhar SA. Short segment pedicle screw instrumentation and augmentation vertebroplasty in lumbar burst fractures: an experience. Eur Spine J. 2008; 17(3):336-41.
} 
3. Venmans A, Klazen CA, Lohle PN, et al. Percutaneous vertebroplasty and pulmonary cement embolism: results from VERTOS II. AJNR Am J Neuroradiol. 2010; 31(8):1451-3.

4. Schmidt R, Cakir B, Mattes T, Wegener M, Puhl W, Richter M. Cement leakage during vertebroplasty: an underestimated problem? Eur Spine J. 2005; 14(5):466-73.

5. Hauck S, Beisse R, Buhren V. Vertebroplasty and kyphoplasty in spinal trauma. Eur J Trauma. 2005; 31:453-63.

6. Röllinghoff M, Siewe J, Eysel P, Delank KS. Pulmonary cement embolism after augmentation of pedicle screws with bone cement. Acta Orthop Belg. 2010; 76(2):269-73.

7. Krueger A, Bliemel C, Zettl R, Ruchholtz S. Management of pulmonary cement embolism after percutaneous vertebroplasty and kyphoplasty: a systematic review of the literature. Eur Spine J. 2009; 18(9):1257-65.

8. Kim YJ, Lee JW, Park KW, et al. Pulmonary cement embolism after percutaneous vertebroplasty in osteoporotic vertebral compression fractures: incidence, characteristics, and risk factors. Radiology. 2009; 251(1):250-9.

9. Choe DH, Marom EM, Ahrar K, Truong MT, Madewell JE. Pulmonary embolism of polymethyl methacrylate during percutaneous vertebroplasty and kyphoplasty. AJR Am J Roentgenol. 2004; 183(4):1097-102.

10. Bernhard J, Heini PF, Villiger PM. Asymptomatic diffuse pulmonary embolism caused by acrylic cement: an unusual complication of percutaneous vertebroplasty. Ann Rheum Dis. 2003; 62(1):85-6.

11. Anselmetti GC, Zoarski G, Manca A, et al. Percutaneous vertebroplasty and bone cement leakage: clinical experience with a new high-viscosity bone cement and delivery system for vertebral augmentation in benign and malignant compression fractures. Cardiovasc Intervent Radiol. 2008, 31(5):937-47.

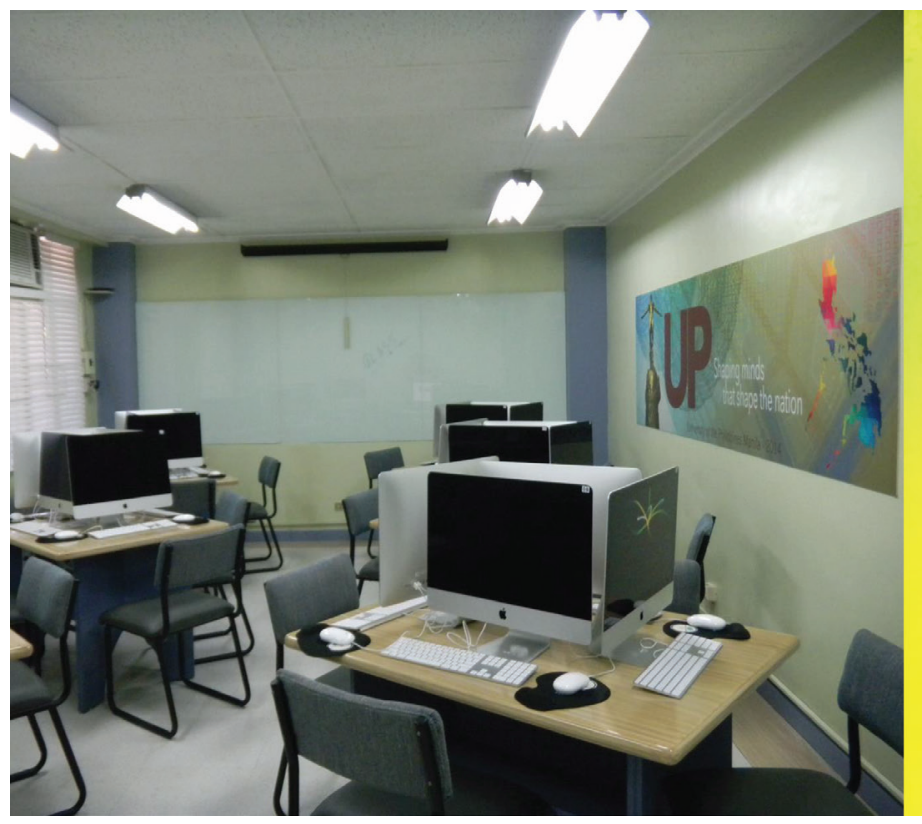

Learning Commons I

The Learning Commons I is a newly renovated space on the first Floor of University Library, UP Manila that brings together library and technology services in an environment that fosters interaction, collaboration and study.

Services:

[ Workstations

28 units of iMac computers are available at the LC I to support teaching, learning and research. The computers are equipped with a variety of productivity software including tools for creating and editing media. The computers maybe used to access the library's subscribed databases and electronic books.

प Media Viewing and Discussion Room

The LCl may be used for small group study, collaborative learning and research, media viewing or individuals/groups to practice presentation. The facility is equipped with LCD projector, viewing screen and whiteboard. Reservation is needed for group study.

[ Printing

Black and white printing is available.

口

Scanning and Digitization

There is one digital scanner in the LC I and can be customized to scan in color or a various page sizes and resolutions. Flash drive is required to save scanned files.

Wireless Network

Tutorials, Guides, Orientation

Library staff may provide one-on-one or group assistance on use of library databases. 